\title{
New Tools for Structural Testing: Piezoelectric Impact Hammers and Acceleration Rate Sensors
}

\author{
C. K. Lee, ${ }^{*}$ C. T. Lin, ${ }^{\dagger}$ C. C. Hsiao, ${ }^{\dagger}$ and W. C. Liaw $^{\dagger}$ \\ National Taiwan University, Taipei, Taiwan 10764, Republic of China
}

\begin{abstract}
Small-size ultra-high-precision mechanical systems demand special testing methodologies, such as a better highfrequency response, a precise impact position, an extremely high repeatability, etc. Utilizing the fact that signals obtained from piezoelectric sensing elements are strongly influenced by the interfacing circuitry, piezoelectric sensors that can be used to measure acceleration rate were developed. Both analytical and experimental results indicate that acceleration rate sensors can detect the arrival of realistic shock earlier than conventional accelerometers can. An ultra-high-precision high-speed piezoelectric impact system with an on-line load cell was also modeled, designed, and built. The sensitivity of this on-line load cell was calibrated by using a standard quartz load cell. This innovative high-speed impact hammer system was found to have a timing accuracy in the range of microseconds and a positioning accuracy in the range of micrometers.
\end{abstract}

\section{Introduction}

A CCOMPANYING high-tech development comes the development of high-performance miniature mechanical systems. These types of devices demand testing methodologies of different merits, such as better high-frequency responses, faster reaction times, etc. Early detection of shock arrival, which typically creates a negative effect on high-performancemechanical systems, can significantly improve structural system performance. In other words, improving the shock arrival detection is the same as improving the high-frequency response of the sensing system. A simple way to improve high-frequency sensor response is to measure the rate of change of the quantity of interest. More specifically, an acceleration rate sensor will have a better high-frequency response than that of accelerometers because differentiation in the time domain equals multiplying $j \omega$ and the frequency spectrum, where $j=\sqrt{ }-1$ and $\omega$ is the angular frequency. However, differentiation in the time domain amplifies the noise, which in turn reduces the accuracy of the rate signal. A method of improving the sensor high-frequency response by measuring the rate of change without deteriorating the signal-to-noise ratio is thus desirable.

On the other hand, modal testing that can reveal the dynamic behavior and execute system identification of today's high-performance mechanical systems is becoming more difficult to perform as impact forces are getting more difficult to apply to testing structures. An impact hammer system with a positioning accuracy in the micrometer range and a timing accuracy in the microsecond range to permit us to perform precise modal or accurate impact testing on miniature mechanical systems is becoming ever more important.

The newly developed sensing and impacting systems, which are the acceleration rate sensor and the high-speed impact hammer system, will be discussed in detail herein.

\section{Acceleration Rate Sensors}

Combining linear piezoelectric theory and Hook's law yields the governing equation for traditional accelerometers. ${ }^{1-6}$ That is, the charge signal $q(t)$ generated from a piezoelectric sensing element is linearly proportional to the acceleration experienced by a piezoelectric sensing element in traditional accelerometers. Because piezoelectric sensing elements are of high-outputimpedance,

Received Nov. 11, 1996; presented as Paper 97-0687 at the AIAA 35th Aerospace Sciences Meeting, Reno, NV, Jan. 6-9, 1997; revision received Feb. 25, 1998; accepted for publication March 1, 1998. Copyright (C) 1998 by the authors. Published by the American Institute of Aeronautics and Astronautics, Inc., with permission.

*Professor, Institute of Applied Mechanics, No. 1, Sec. 4, Roosevelt Road. Senior Member AIAA.

${ }^{\dagger}$ Graduate Research Assistant, Institute of Applied Mechanics, No. 1, Sec. 4, Roosevelt Road. the externally obtained signals from a piezoelectric sensing element are strongly influenced by the interfacing circuitry used. It has been identified that changing the charge amplifier typically used in traditional piezoelectric accelerometers to a current amplifier is the equivalent of time differentiation. ${ }^{4,6}$ In other words, the rate signal can be obtained by measuring the current signal $i(t)=\mathrm{d} q(t) / \mathrm{d} t$ without using a mathematical differentiation process. This is the basic concept of the interfacing circuit used for the piezoelectric acceleration rate sensor. ${ }^{6}$

Considering a realistic impact force that can appear in highperformance structural systems, the impact force amplitude is initially zero and increases rapidly with time. That is,

$$
f\left(t \rightarrow 0^{+}\right)=f\left(0^{+}\right)+\dot{f}\left(0^{+}\right) t+O\left(t^{2}\right)
$$

where $f\left(0^{+}\right)$typically equals zero for a realistic impact force due to the shock arrival time constraintin a mechanical system. Physically, any shock excitation source has a finite rising time such as a sudden hertz impact. Consider the contribution of a realistic impact force on a simple mass-spring system $m \ddot{x}+k \dot{x}=f(t)$, where $m$ and $k$ are the mass and stiffness of the mass-spring system, respectively, and under the initial conditions $x\left(0^{-}\right)$and $\dot{x}\left(0^{-}\right)=0$. The response of this spring-mass system can be shown to be

$$
x\left(t \rightarrow 0^{+}\right)=\lim _{t \rightarrow 0^{+}} \frac{\dot{f}(t)}{6 m} t^{3}+O\left(t^{4}\right)
$$

where $O(\cdot)$ denotes the big- $O$ notation. Differentiating Eq. (2) several times with respect to time yields the governing equations for acceleration

$$
\ddot{x}\left(t \rightarrow 0^{+}\right)=\lim _{t \rightarrow 0^{+}} \frac{\dot{f}(t)}{m} t+O\left(t^{2}\right)
$$

and the acceleration rate

$$
\frac{\mathrm{d} \ddot{x}}{\mathrm{~d} t}\left(t \rightarrow 0^{+}\right)=\lim _{t \rightarrow 0^{+}} \frac{\dot{f}(t)}{m}+O(t)
$$

From the preceding equations, it is clear that the acceleration rate has a finite value at $t \rightarrow 0$, whereas the accelerometer output signal equals zero as $t \rightarrow 0$. Thus, theoretically, the acceleration rate can be detected earlier than acceleration. From this viewpoint, the acceleration rate is a better sensing variable than acceleration itself for shock detection or even shock control.

An impact testing experimental setup as shown in Fig. 1 was created to examine the performance of the acceleration rate sensor vs that of the accelerometer. The acceleration rate sensor was created by replacing the Endevco Model 22 accelerometer ${ }^{7}$ charge amplifier interfacing circuit with a Keithley 427 current amplifier. ${ }^{8}$ The 


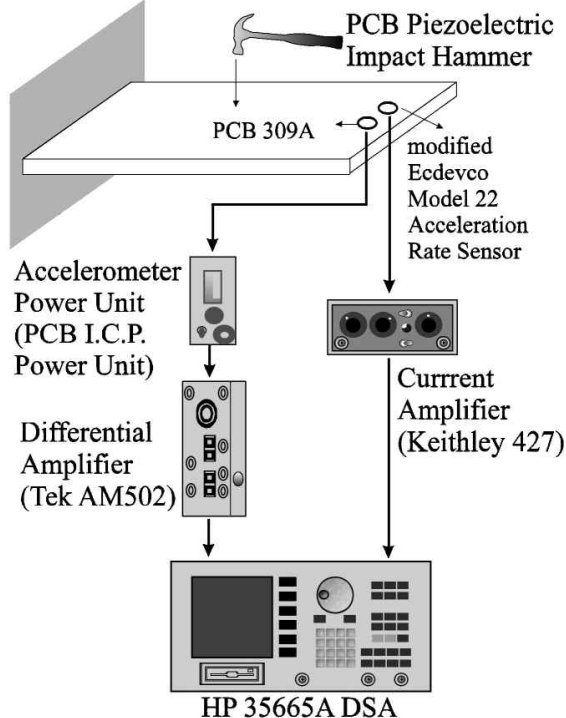

Fig. 1 Impact testing setup for the acceleration rate sensor and the accelerometer.

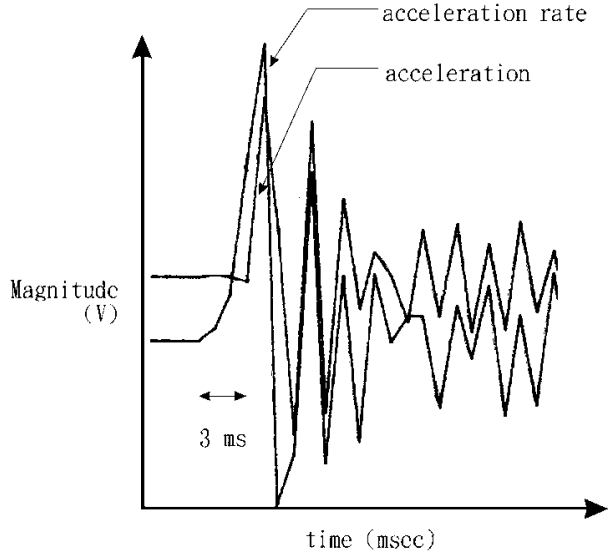

Fig. 2 Comparison of shock arrival between the acceleration rate sensor and the accelerometer.
PCB309A accelerometer, ${ }^{9,10}$ which has a built-in interfacing circuit and was connected to an external measurement instrument by a voltage amplifier, was used as the comparison sensor. Both sensors were placed at the same longitudinal position of the testing beam structure. The bandwidth of the voltage amplifier and the current amplifier was adjusted to be identical. Finally, both the accelerationrate signal and acceleration signal were connected to a Hewlett-Packard $35665 \mathrm{~A}$ dynamic signal analyzer ${ }^{11}$ for an arrival time comparison.

The results shown in Fig. 2 indicate that the acceleration rate sensor detects shock arrival earlier than the accelerometer when the impact hammer generates a mechanical impulse to the beam. In Fig. 2, the upper trace is the acceleration rate signal and the bottom trace is the acceleration signal. It is clear from the data that the measured shock arrival time for the acceleration rate sensor was $156.25 \mathrm{~ms}$ and for the accelerometer was $159.179 \mathrm{~ms}$. Thus, a total of 3.0-ms difference in shock arrival detection was noted.

The linear spectrum between the acceleration rate sensor and the accelerometer was also generated to make the comparison. To create the acceleration spectrum from the acceleration rate signal, a pseudointegration that is equal to dividing the acceleration rate spectrum by $j \omega(j=\sqrt{ }-1$, and where $\omega$ is the angular frequency) was performed. It is clear from Fig. 3 that the spectrum between the pseudointegratedacceleration rate signal obtained by integrating the modified Endevco Model 22 accelerometer signal agrees well with the accelerometer spectrum. Note that the gain difference between the two traces is of no significant importance due to the arbitrary gain setting of the two sensors. The difference that appears between the two traces for a frequency in the $0-80 \mathrm{~Hz}$ range can be attributed to the fact that acceleration rate sensors naturally emphasize a higherfrequency portion of the spectrum than do accelerometers. In other words, an acceleration rate sensor is more naturally tuned to higherfrequency detection than an accelerometer.

One thing that should be noted is that the aforementioned piezoelectric acceleration rate sensor essentially operates the piezoelectric sensing element under the charge mode. In comparison, the PCB309A accelerometer mentioned earlier was running under the voltage mode due to the interfacing circuitry used. There are many different performance merits for piezoelectricsensing elements running under voltage mode vs charge mode. For example, temperature stability is of ultimate importance if the piezoelectric sensing elements are to be used in various temperature conditions. It is known that piezoelectric ceramics such as PZT (lead, zirconate, titanate) are more stable in temperature when they are used in a charge mode. Adapting these types of materials to the situation mentioned

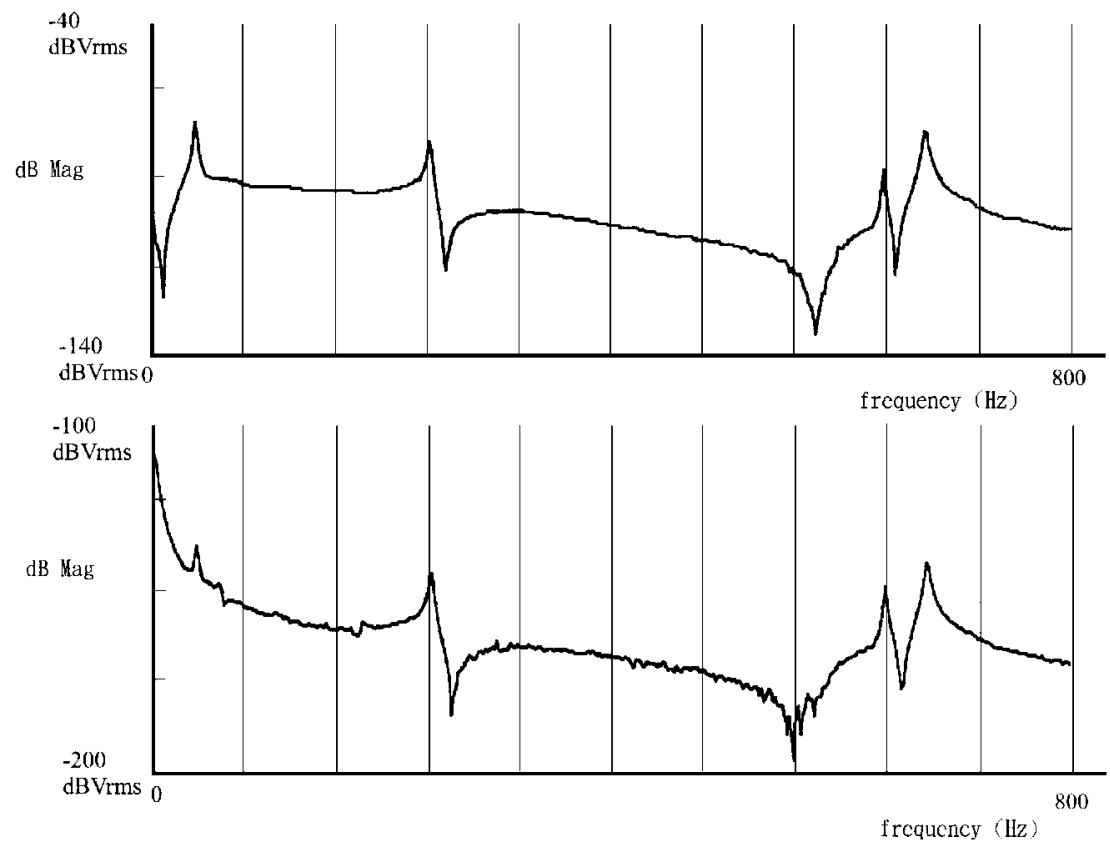

Fig. 3 Linear spectrum of an accelerometer signal (top trace) and pseudointegrated acceleration rate sensor signal (bottom trace). 
earlier, which used the current amplifier to interface the piezoelectric sensing element, can generate an acceleration rate sensor of hightemperature stability. On the other hand, a piezoelectric polymer such as polyvinylidene fluoride $\left(\mathrm{PVF}_{2}\right)$ is more temperature stable when it is used in the voltage mode. Anyone who intends to adopt the current amplifier circuitry concept for piezoelectric polymerictype sensing elements should be aware that high-temperature stability is traded for differentiation noise immunity.

Another thing that should be noted is that the time difference between shock arrival detected by accelerometers and by acceleration rate sensors will depend on factors such as specimen conditions, interfacing circuit bandwidth, etc., to name a few. Nevertheless, both the theoretical and analytical results presented earlier show that

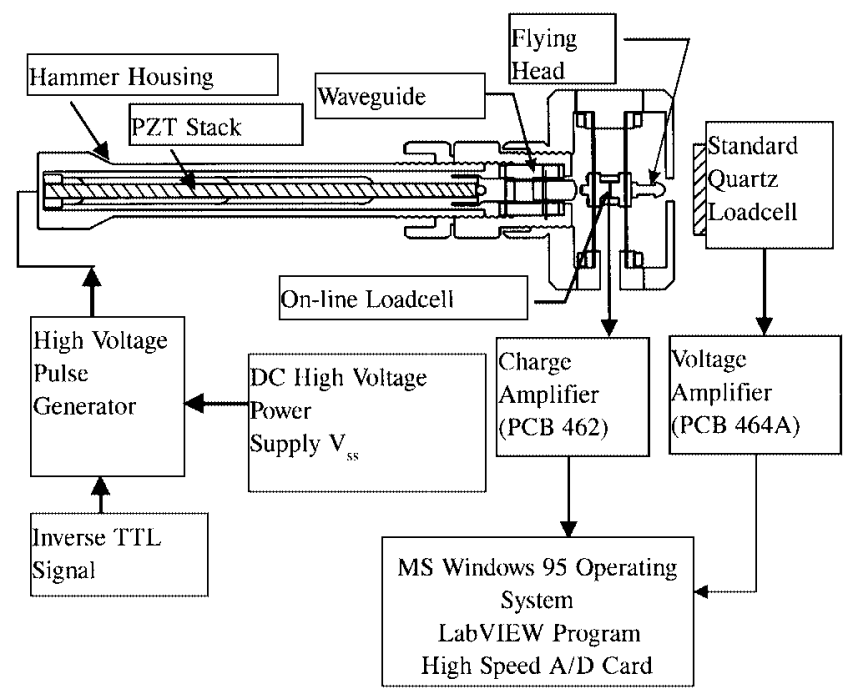

Fig. 4 Schematic of the impact hammer system design/calibration.

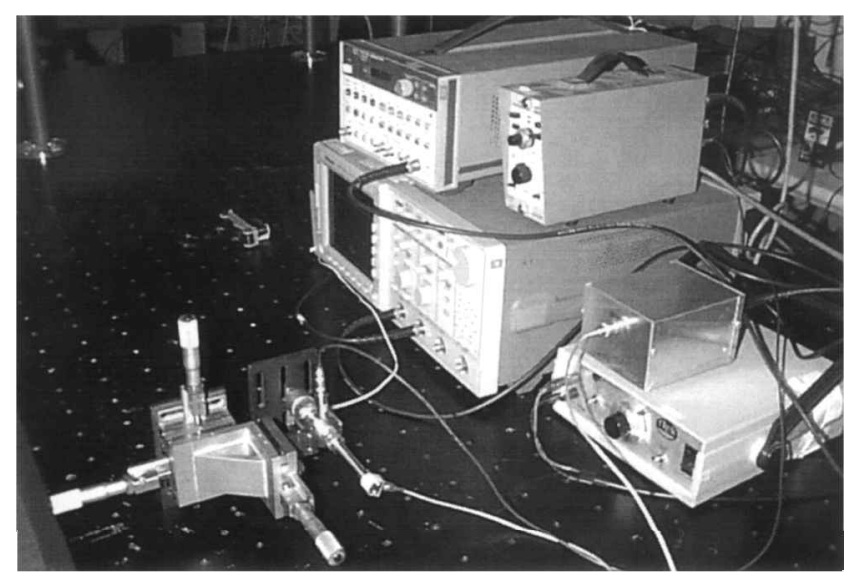

Fig. 5 Photo of the impact hammer system design/calibration. acceleration rate sensors detect shock earlier than the accelerometers do.

\section{Precision Impact Hammer System}

A piezoelectric material is characterized as having a large force exerted but with very little deformation. Many mechanically magnifying mechanisms, which are all based on the leveling principle to magnify motion, have been developed over the years to overcome such a problem. ${ }^{12-18}$ A completely different concept, which is based on a waveguide design to transform the blocking force of piezoelectric materials into a free-flying motion, was created by Chang and Wang ${ }^{19,20}$ to achieve large motion for piezoelectrically driven mechanisms. This mechanism was later improved by Lee and $\mathrm{Wu},{ }^{21}$ and $\mathrm{Wu}$ and Lee, ${ }^{22}$ who added an on-line load cell into a free-flying object to detect impact force as well as to create a piezoelectric impact hammer system. The optimization process and many design modifications to the aforementioned system will be discussed in detail herein. The basic layout of our newly developed ultra-high-precision high-speed impact hammer system is shown in Figs. 4-6. To optimize the waveguide design used in this paper and to understand the propulsion process better, this driving mechanism will be modeled herein.

As the capacitance value of the piezoelectric rod is in the range of $1.25 \mu \mathrm{F}$, the normal power supply will not be able to provide enough current to drive the piezoelectric rod. The driving circuit used is based on a capacitor bank discharge concept as shown in Fig. 7. That is, a large amount of charge was stored within a set of high-value high-voltagecapacitors first, and then all the charge was released to drive the piezoelectric rod. The typical voltage waveform generated is shown in the upper trace of Fig. 8. The inverse transistor-transistor logic (TTL) $(0$ to $-5 \mathrm{~V})$ signal shown in the bottom trace of Fig. 8 was used to initiate the whole discharging process so as to send a high-voltage pulse into the piezoelectric rod. The dc power supply $V_{s s}$ was set at $150 \mathrm{~V}$ and was used to charge the high-value highvoltage capacitor. The TTL time width $T_{b}$ is used to determine the voltage level that the piezoelectric stack should be charged. All in all, the newly designed driving circuit charges the piezoelectric stack with one time constant and discharges with a different time constant.

The driving mechanism of this newly developed precision impact hammer system was modeled by using the one-dimensional piezoelectric elastic wave theory to obtain the governing equation (see Fig. 9)

$$
\frac{\partial^{2} u}{\partial x^{2}}=\frac{1}{c^{2}} \frac{\partial^{2} u}{\partial t^{2}}
$$

where $c=\sqrt{ }\left(Y_{p} / \rho\right)$ represents the wave velocity, and $Y_{p}$ and $\rho$ are Young's modulus and the density of the piezoelectric material, respectively. The initial conditions $u(x, 0)=0$ and $\partial u(x, 0) / \partial t=0$ and the boundary conditions $u(x=0, t)=0$ at the fixed end and $\partial u(l, t) / \partial x=d E(t)-\left[k_{s} u(t)+k_{s} u_{0}+m_{0} \partial^{2} u(t) / \partial t^{2}\right] / A Y_{p}$ at the preload end are needed to model the impact hammer system. In addition, $l$ is the length of the piezoelectric rod, $A$ is the crosssectional area of the piezoelectric rod, $d$ and $e$ are the piezoelectric strain/charge and piezoelectric stress/charge constants, $E(t)$ is the

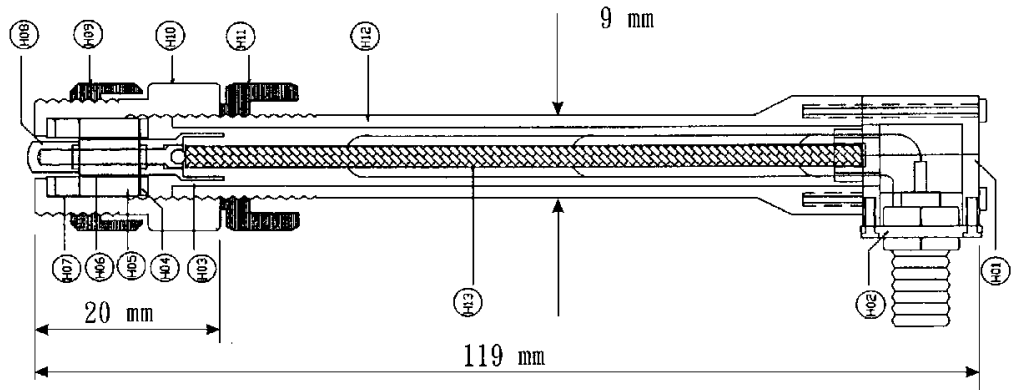

H01:connecter H04:screw holder H07:screw holder H10:pre-load top housing H11:lock 2
$\mathrm{H} 02$ : cover plate

H05:spring spacer

rwaveguide

H09:lock

H12:PZT rod housing

Fig. 6 Schematic of the impact hammer. 


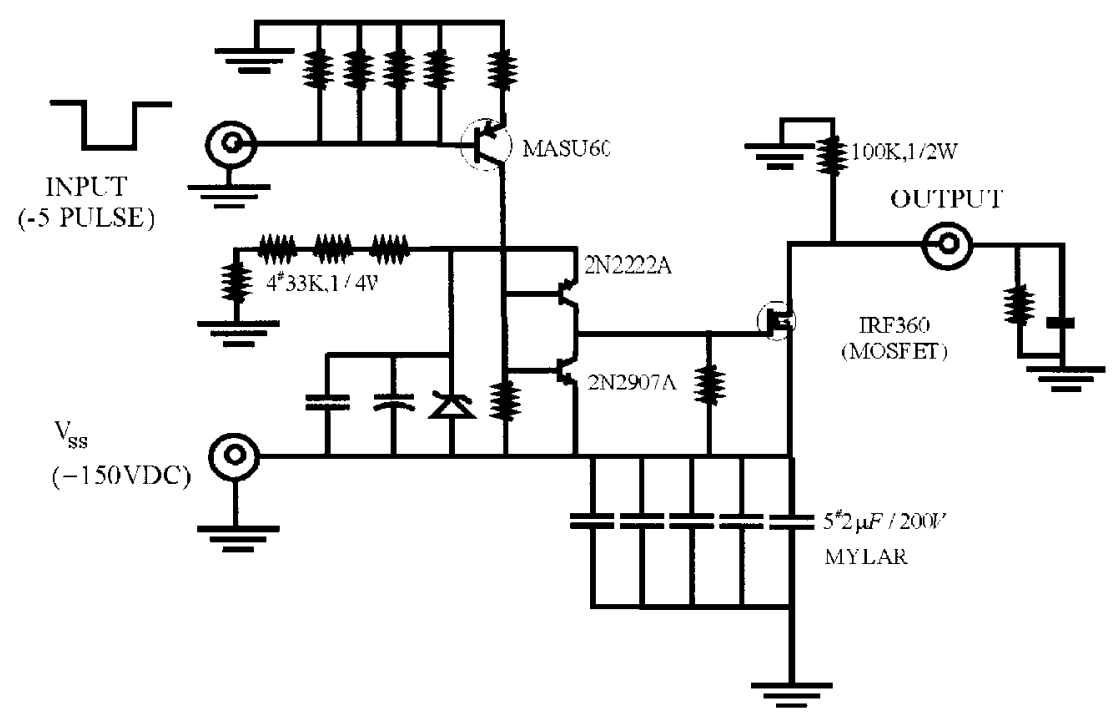

Fig. 7 Design circuit for charging PZT stacks.
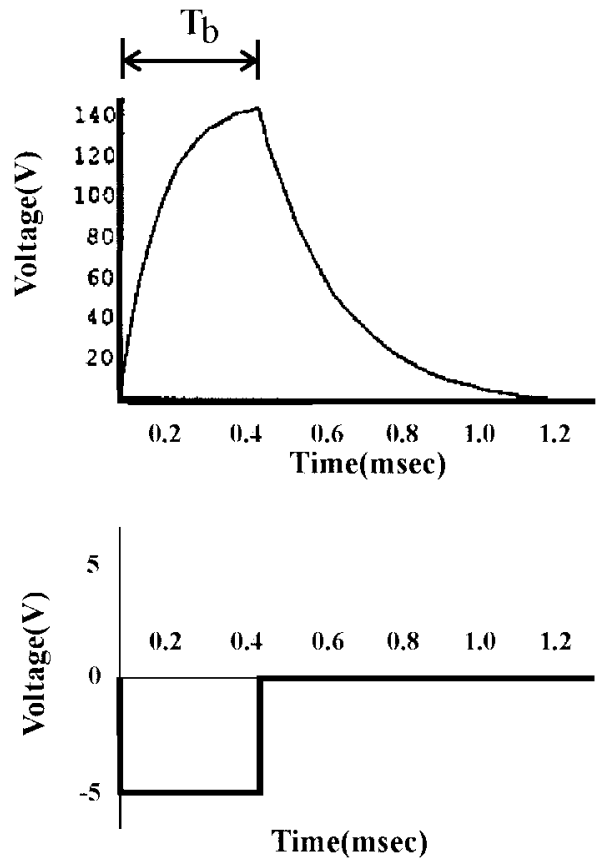

Fig. 8 Typical waveform generated by the high-voltage pulse generator.

applied electric field, $u_{0}$ is the preload displacement, $m_{0}$ is the added mass, and $k_{s}$ is the equivalent to the spring preload.

Choosing $T=t c / l, X=x / l, U(X, T)=u(x, t) / l, U_{0}=u_{0} / l$, $M=m_{0} / \rho A l$, and $K=k_{s} l / A Y_{p}$, Eq. (5) can be transformed into a nondimensional form:

$$
\frac{\partial^{2} U}{\partial X^{2}}=\frac{\partial^{2} U}{\partial T^{2}}
$$

with the initial conditions $U(X, 0)=0$ and $\partial U(X, 0) / \partial T=0$ and the boundary conditions $U(X=0, T)=0$ at the fixed end and $\partial U(X=1, T) / \partial x=D(T)-K U_{0}-K U(X=1, T)-M \partial^{2} U$ $(X=1, T) / \partial T^{2}$ at the preloadend. In addition, the nondimensional parameter $D(T)=d E(T)$ represents the electrical field effect of the piezoelectric column, and the nondimensionalized $K$ represents the relative stiffness between the preload spring $k_{s}$ and the piezoelectric $\operatorname{rod} A Y_{p} / l$. All nondimensional variables can be recognized by the use of capital letters that specify them. Taking a Laplace transform on Eq. (6) and on the initial and boundary conditions yields

$$
\frac{\partial^{2} \tilde{U}(X, S)}{\partial X^{2}}-S^{2} \tilde{U}(X, S)=0
$$

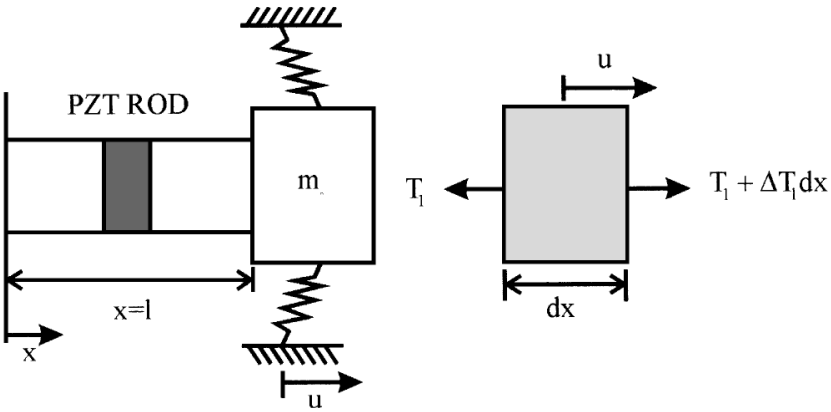

Fig. 9 Free body diagram of the piezoelectric rod driving system.

with the Laplace transformed boundary conditions: $\tilde{U}(X=0, S)=$ 0 , and $\partial \tilde{U}(X=1, S) / \partial X+\left(K+M S^{2}\right) \tilde{U}(X=1, S)=\tilde{D}(S)-$ $U_{0} K / S$. The solution of displacement in the Laplace transformed $S$ space can then be solved. Taking the inverse Laplace transform yields the following solution:

$$
\begin{aligned}
& U(X, T)=\frac{1}{2 \pi j} \int_{\gamma-j \infty}^{\gamma+j \infty} \tilde{U}(X, S) e^{S T} \mathrm{~d} S \\
& \quad=\frac{1}{2 \pi j} \int_{\gamma-j \infty}^{\gamma+j \infty} \frac{\left(K U_{0}-\tilde{D} S\right)}{S F(S)} \sinh (S X) e^{S T} \mathrm{~d} S
\end{aligned}
$$

where $F(S)=S \cosh (S)+\left(M S^{2}+K\right) \sinh (S), \tilde{D}(S)=S\left\{D_{0}(1-\right.$ $\left.e^{-T_{b} S}\right) / S-D_{0}\left[1-e^{-\left(S+1 / \tau_{1}\right) T_{b}}\right] /\left(S+1 / \tau_{1}\right)+D_{0} e^{-T_{b} S\left[1-e^{-T_{b} / \tau_{1}}\right]} /(S+$ $\left.\left.1 / \tau_{2}\right)\right\}$, and $\tilde{D}(S)$ and $D(T)$ are the Laplace transformed pair. More specifically, the high-voltage pulse generated can be viewed as the combination of a charge process with time constant $\tau_{1}$ and a discharge process with time constant $\tau_{2}$. The symbolic programming language MACSYMA ${ }^{23}$ was used to perform a Pade approximation to expand the denominatorof the integrandof Eq. (8). From there, the residual theorem was then used to evaluate the expanded equation.

Once the wave propagation behavior was modeled within the piezoelectric rod and the preload mechanism, the interaction between the waveguide and the flying head was simulated by using a Hopkinson bar model. ${ }^{24}$ For the case where $\partial u / \partial x<0$, the interface between the flying head and the waveguide is compressed, and the wave is transmitted from the waveguide to the flying head. For the case where $\partial U / \partial x \geq 0$, the interface is under tension, and the flying head is considered to be separated from the waveguide. The force applied on the flying head under various $K$ computed by using the Hopkinson bar model can be shown in Fig. 10.

To verify theoretical predictions and to examine the performance of this newly developed piezoelectric impact hammer system, an on-line load cell (OLC) was embedded into the flying head to detect the impact force. The setup shown in Fig. 4 was used for the impact 


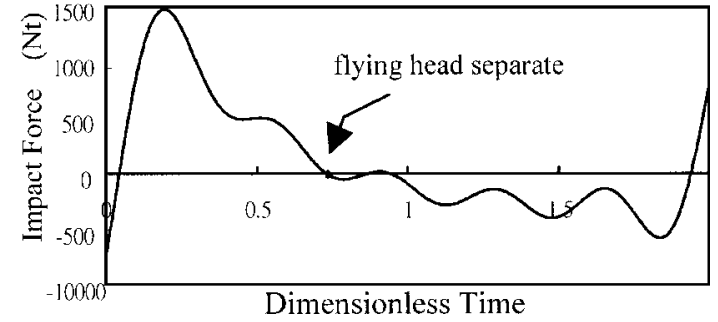

a) $K=0.01$ and $M=0.4$

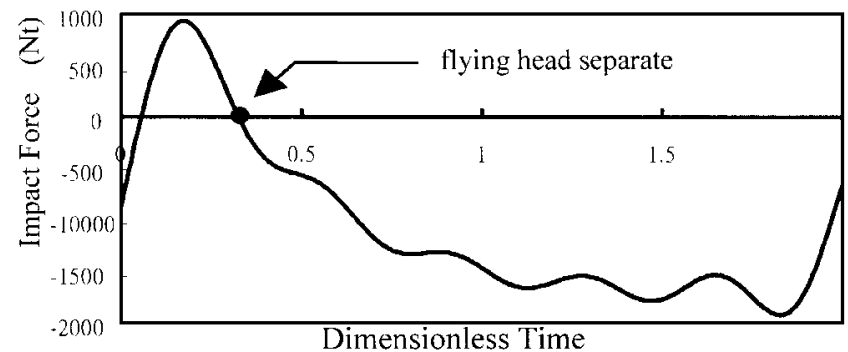

b) $K=0.1$ and $M=0.4$

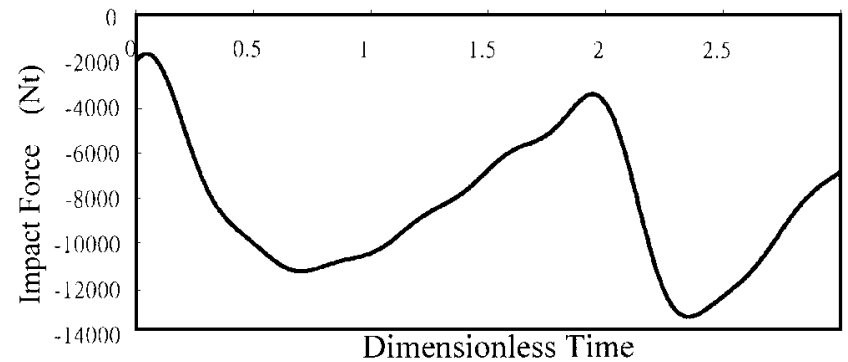

c) $K=1$ and $M=0.4$

Fig. 10 Influence of different $K$ on the transmitted force pulse computed by using the Hopkinson bar concept.
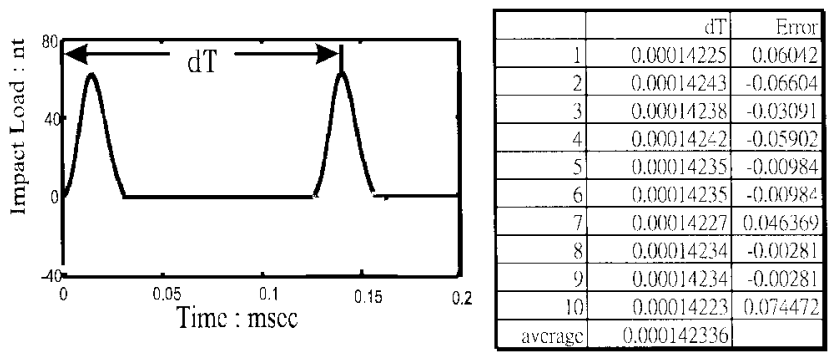

Fig. 11 Timing accuracy of the impact hammer system.

hammer calibration and for performance evaluation. A PCB model $209 \mathrm{~A}^{25}$ standard quartz load cell (SLC) of $8.1-\mathrm{mV} / \mathrm{g}$ sensitivity was used as the calibration sensor. The graphic user interface software LabVIEW $^{26}$ was used to communicate with a Gage card ${ }^{27}$ of 250 MHz sampling rate to record the signal generated from the OLC and the SLC. The sensitivity of the OLC can be obtained by comparing the signal between the OLC and the SLC.

The impact location repeatability and timing accuracy of the impact hammer system were also examined. A total of 10 sets of pulses generated by the OLC were recorded in Fig. 10 by use of a Gage card. All of these signals were triggered by the inverse TTL signal used to initiate the piezoelectric driving mechanism. The flying time difference $\mathrm{d} T$ between the first two pulses was measured to be far less than $1 \mu$ s (see Fig. 11). The impact location accuracy was examined by checking the center impact mark location difference for a single impact and for a set of 10 consecutiveimpacts under the same condition and at the same location. The impact location accuracy was found to be in the range of $1 \mu \mathrm{m}$.

The parameters used in our experimental results are $l=72 \mathrm{~mm}$, and where $A$ is $2 \times 3 \mathrm{~mm}, Y_{p}=5.5 \times 10^{10} \mathrm{~N} / \mathrm{m}^{2}$, and $k_{s}=6.48 \times$ $10^{4} \mathrm{~N} / \mathrm{m}$. The $K$ value used was $K=k_{s} / A Y_{p} \approx 0.014$. For typical experimental results, the value of $K$ is in the range of $0.01-0.1$. As

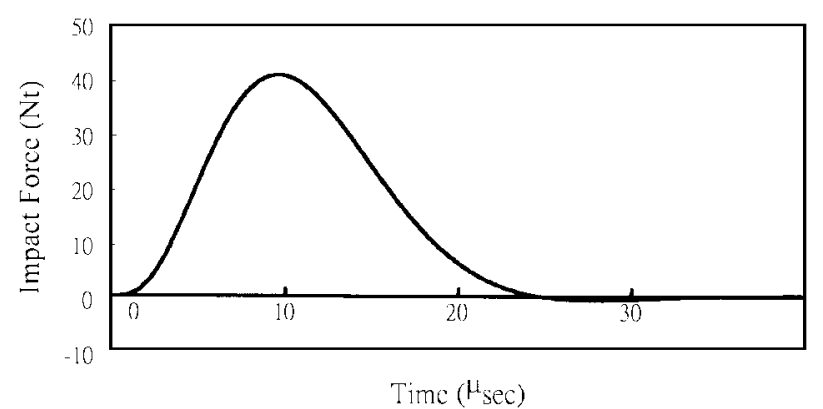

a) $K=0.01$ and $M=0.4$

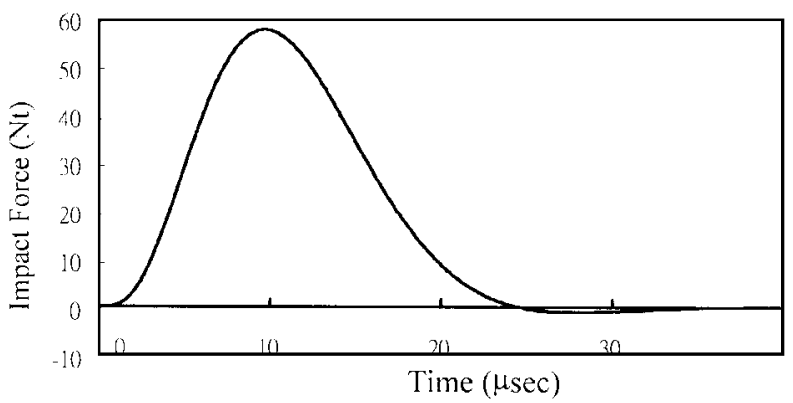

b) $K=0.1$ and $M=0.4$

Fig. 12 Digitally filtered piezoelectric force signal for the case.

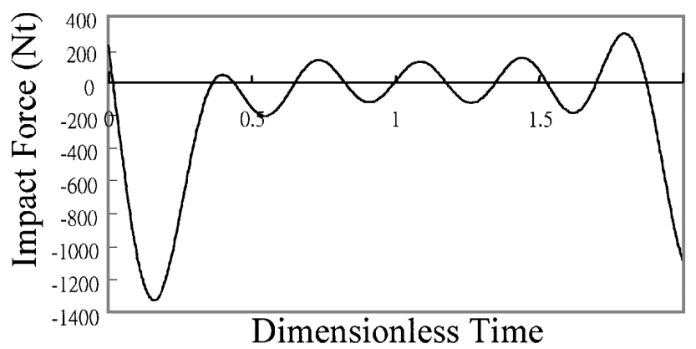

a) $K=0.1$ and $M=0.005$

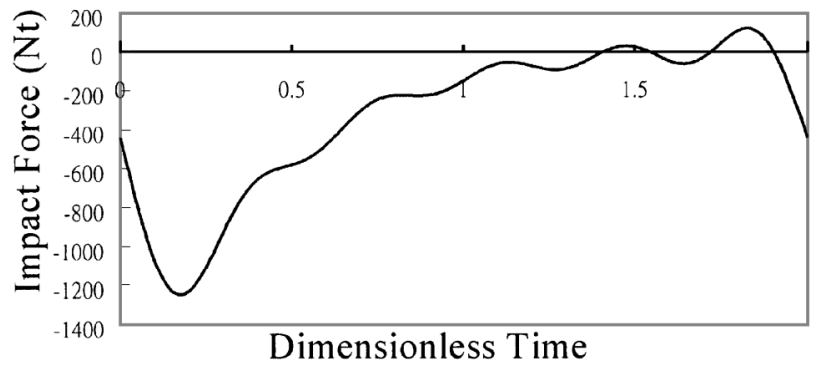

b) $K=0.1$ and $M=0.4$

Fig. 13 Influence of added mass.

the band width of the experimental data was limited at $1-50 \mathrm{kHz}$ by a second-orderButterworth filter, ${ }^{28}$ the theoretical predictions shown in Figs. 10a and $10 \mathrm{~b}$ need to be modified by using the same digital filter (Fig.12). The magnitude and impact time for the driving impulse obtained from our experiments (first impulse of Fig. 10a) and theoretical predictions (Fig. 12) agree quite well. It is also clear from Fig. 10 that a larger $K$ will induce a larger impulse force for the range where $K$ is between 0.01 and 0.1 . It is also clear from Fig. 10 that the interaction on the interface will transform from thrust to compression as the $K$ value passes through 0.1 . Considering the theoretical prediction purely from a wave propagation viewpoint, the value of $K$ can be viewed to represent the relative acoustical impedance between the waveguide and the flying head. In this viewpoint $K=1$ and $M=0$ should be the transitional points concerned as the reflected wave will have a $180-\mathrm{deg}$ phase shift when the interface is met. The discrepancy on the transitional $K$ value lies on the effect of the added mass. It is clear from Fig. 13 that the added mass will make the transitional $K$ value appear smaller than $K=1$. 
Also note that the interface between the waveguide and the flying head is not a rigid body and the stress-concentration phenomenon can be predicted when $K>0.1$ and $M=0.4$. Thus, the Hopkinson bar model is considered unsuitable to model this type of impact hammer system for the case where $K>0.1$ and $M=0.4$.

\section{Conclusions}

To pursue the increasingly more demanding mechanical testing tasks of today, an accelerationrate sensor and an ultra-high-precision impact hammer system were developed. Using a current amplifier to interface the high-output impedance piezoelectric sensing element, an acceleration rate sensor, which detects shock arrival earlier than that of an accelerometer, was created. Because of the rate nature of this acceleration rate sensor, this newly created acceleration rate sensor not only can detect shock arrival earlier but also can be naturally tuned to higher-frequency detection.

By using the waveguide concept, the blocking force of piezoelectric materials was transformed into an impact hammer system. In addition, the Hopkinson bar concept was adopted to examine the possibility of optimizing this impact hammer system. The experimental data indicate that this piezoelectric impact hammer has a timing accuracy in the range of microseconds and a positioning accuracy in the range of micrometers. The short impact time achieved by the impact hammer also warrants a higher-frequency excitation achievable by the impact hammer.

Combining the high-frequency feature of both the acceleration rate sensor and the impact hammer system, these two newly developed testing systems make testing of miniature mechanical structures feasible.

\section{Acknowledgment}

The results presented in this paper were partially supported by the National Science Council of Taiwan, Republic of China, under Contract NSC 85-2612-E-002-056.

\section{References}

${ }^{1}$ Lee, C. K., "Piezoelectric Laminates for Torsional and Bending Modal Control: Theory and Experiment," Ph.D. Dissertation, Dept. of Theoretical and Applied Mechanics, Cornell Univ., Ithaca, NY, 1987.

${ }^{2}$ Uchino, K., "Electrostrictive Actuators: Materials and Applications," Ceramic Bulletin, Vol. 65, No. 4, 1986, pp. 647-652.

${ }^{3}$ IEEE Standard on Piezoelectricity, ANSI/IEEE Std. 176-1987, Inst. of Electrical and Electronics Engineers, New York, 1987, pp. 1-54.

${ }^{4}$ Lee, C. K., and O'Sullivan, T. C., "Piezoelectric Strain Rate Gages," Journal of the Acoustical Society of America, Vol. 90, No. 2, 1991, pp. 945-953.

${ }^{5}$ Lee, C. K., "Piezoelectric Laminates: Theory and Experiments for Distributed Sensors and Actuators," Intelligent Structural Systems, ed. by H. S. Tzou, and G. L. Anderson, Kluwer Academic, Dordrecht, The Netherlands, 1992, pp. 75-167.
${ }^{6}$ Lee, C. K., Lin, C. T., Hsiao, C. C., Kuo, C. C., and Lin, J. Y., "Innovative Piezoelectric Acceleration Rate Sensors," Proceedings of the AIAA/ASME/ASCE/AHS/ASC 37th Structures, Structural Dynamics, and Materials Conference, Reston, VA, April 1996, pp. 1035-1042.

7 “Picoman 22 Accelerometer Manual," Endevco Corp., 30700 Rancho Viejo Road, San Juan Capistrano, CA 92675, May 1990, pp. 1-10.

8"Model 427 Current Amplifier Manual," Keithley Instrument, Inc., Instrument Division, 28775 Aurora Road, Cleveland, OH 44139, 1991, pp. $1-7$.

9"Vibration and Shock Sensor Selection Guide," PCB Piezotronics, Inc., 3245 Walden Avenue, Depew, NY 14043-2495, 1992, p. 93.

10“Catalog G-500," PCB Piezotronics, Inc., 3245 Walden Avenue, Depew, NY 14043-2495, 1993, p. 134.

11 “HP 35665 User's Manual," Hewlett-Packard Co., 8600 Soper Hill Road, Everett, WA 98205-1298, 1993, pp. 4.1-4.297.

${ }^{12}$ Takahashi, S., "Multilayer Piezoelectric Ceramics Actuators and Their Applications," Japan Journal of Applied Physics Supplemental, Vol. 24, No. 2, 1985, pp. 41-45.

${ }^{13}$ Fukui, I., Hamatsuki, T., Yano, T., Sato, E., and Inui, O., "Impact Printer Head Capable of Printing a Dot at a Distance Narrower than a Thickness of a Printer Unit," U.S. Patent 4,589,786, May 20, 1986.

${ }^{14}$ Kitagawa, K., "Printing Mechanism for Dot Matrix Printers," U.S. Patent No. 4,613,241, Sept. 23, 1986.

${ }^{15}$ Ota, T., Uchikawa, T., and Mizutani, T., "Printing Flight Hammer Using Multi-Layer Piezoelectric Actuator," Japan Journal of Applied Physics Supplemental, Vol. 24, No. 2, 1985, pp. 41-45.

${ }^{16}$ Sakurai, K., Suga, M., and Inoue, T., "Investigations on an Ultrasonic Piezoelectric Printer," Japan Journal of Applied Physics Supplemental, Vol. 26, No. 1, 1987, pp. 141-143.

${ }^{17}$ Sakamann, W., "Matrix Printer with Piezoelectrically Driven Printing Needles," U.S. Patent 4,193,703, March 18, 1980.

${ }^{18}$ Asano, H., "Stacked Piezoelectric Ceramics Displacement Magnifying Devices," U.S. Patent No. 4,703,215, Oct. 27, 1987.

${ }^{19}$ Chang, P. S. H., and Wang, H. C., "A High Speed Impact Actuator Using Multilayer Piezoelectric Ceramics," Sensors and Actuators, Vol. 24, No. 3 , Sept. 1990, pp. 239-244.

${ }^{20}$ Chang, P. S. H., and Wang, H. C., "Matrix Printer Actuator," U.S. Patent No. 5,046,872, Sept. 1991.

${ }^{21}$ Lee, C. K., and Wu, T. W., "Piezoelectric Hammer with On-Line Loadcell," IBM Technical Disclosure Bulletin, Vol. 36, No. 3, 1993, pp, 175178.

${ }^{22}$ Wu, T. W., and Lee, C. K., "Micro-Impact Technique and Its Applications," Journal of Materials Research, Vol. 9, No. 3, 1994, pp. 787-804.

23"Reference Manual," v. 14, MACSYMA, Inc., 8 New England, Executive Park East, Burlington, MA 01803, April 1995.

${ }^{24}$ Graff, K. F., Wave Motion in Elastic Solids, Dover, New York, 1991, pp. 130-133.

25“Transducer Instrumentation: Model 209A Force Transducer," PCB Piezotronics, Inc., 3245 Walden Avenue, Depew, NY 14043-2495, 1994.

26"LabVIEW for Windows," National Instruments Corp., 6504 Bridge Point Parkway, Austin, TX 78730-5039, Dec. 1993.

27 “'Technical Reference and User's Guide for CompuScope 250," Gage Applied Sciences, Inc., Montreal, PQ, Canada, Aug. 1995, pp. 4.1-4.15.

${ }^{28}$ Jackson, L. B., Digital Filter and Signal Processing, Kluwer Academic, Dordrecht, The Netherlands, 1986. 